\title{
Construction of a Kösters Double-Image Prism
}

\author{
J. B. Saunders
}

\begin{abstract}
An interferometric method is deseribed for use in constructing and adjusting doubleimage prisms of the Kösters type. The faces of the prisms form the elements of several interferometers that are used for testing and for making adjustments during construction. The prisms are cemented together and are quite stable. The precision attainable in the adjustments approaches interferometric perfection.
\end{abstract}

\section{Introduction}

The Kösters double-image prism ${ }^{1}$ forms an important element in several types of interferometers However, there is little information in the literature on its construction. Private correspondence with other laboratories indicates that the components of previously constructed prisms have been separated by thin films of oil and held in adjustment by their housing. This manner of assembly seems unstable, necessitating the frequent use of fine adjustments, thereby increasing difficulties in applications. A technique is described whereby these compound prisms, and modifications ${ }^{2}$ of them, can be accurately constructed and cemented with hardened cements that favor stability and permanence of calibration.

\section{Construction of Double-Image Prism}

\subsection{Prism Components}

The compound prism is made from a pair of nearly identical prisms. For ease of discussion the angles and faces of the prisms are labeled as shown in figure 1 . A prism that is a little more than twice as long as the final prism is ground and polished. The angle $\alpha$ is adjusted, by grinding the original prism to approximately the desired value, and the adjacent faces $\mathrm{BB}^{\prime}$ and $\mathrm{CC}^{\prime}$ are then polished to a high degree of planeness. Face $\mathrm{AA}^{\prime}$ may be cloth polished, as its finish is of little importance at this stage. Angles $\beta$ and $\gamma$ are not critical for precise adjustment of these components. These angles and faces $\mathrm{A}$ and $\mathrm{A}^{\prime}$ will be finished by fine polishirg, using interference of light for testing them, after the thickness of the cement and relative position of the prisms have been adjusted and the cement has hardened.

The prism shown in figure 1 is sawed into two prisms of equal length by cutting through a section parallel to the ends and midway between them.

\footnotetext{
${ }^{1} \mathrm{~W}$. Kösters, Interferenzdoppelprisma fur Messzwecke, Deutsches Reich Patentschrift 595211 (1934)

2 J. B. Saunders, Inverting interferometer, J. Opt. Soc. 45, 133 (1955).
}

This produces two prisms ( $\mathrm{ABC}$ and $\mathrm{A}^{\prime} \mathrm{B}^{\prime} \mathrm{C}^{\prime}$ ) of equal angles, which are identified from the designations of their faces. The prisms then receive semitransparent coats of aluminum on the entire area of face $\mathrm{B}^{\prime}$ and the lower one-third (toward the base) of face $\mathrm{B}$. They are then cemented together (face B to $\mathrm{B}^{\prime}$, as shown in fig. 2a) with no attempt toward paralleling the film of cement or adjusting the prisms.

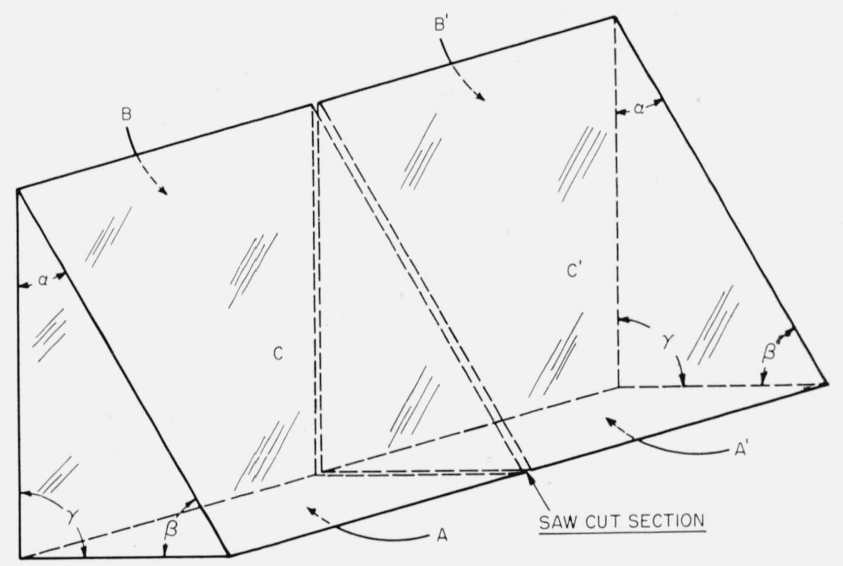

Figure 1. Components of a Kösters double-image prism.
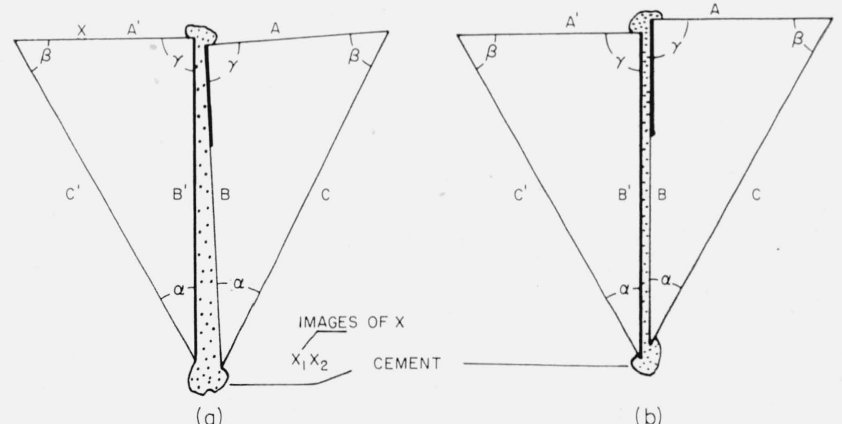

(b)

Figure 2. Cemented prism before it is adjusted. 


\subsection{Optical Cement}

Any optical cement that can be worked as a liquid, becoming hard at ambient temperatures, can be used in these prisms. Canada balsam has been used quite successfully. Its hardness is adjusted, by distillation of volatile constituents, to a consistency considered proper, by an experienced optician, for cementing lenses. The refractive index and dispersion of the sample used here were quite similar to the glass of the prisms. ${ }^{3}$ This combination of cement and glass practically eliminates reflection and refraction at the uncoated parts of surface B. The cement effectively becomes a part of prism $\mathrm{ABC}$, and the acute angle between $\mathrm{B}$ and $\mathrm{C}$ disappears optically and becomes effectively the variable angle between faces $\mathrm{B}^{\prime}$ and $\mathrm{C}$. Figure 2, b, shows the prism after the film of cement has been adjusted parallel and the vertex of angle $\alpha$ of prism $\mathrm{A}^{\prime} \mathrm{B}^{\prime} \mathrm{C}^{\prime}$ has been adjusted to lie in the plane of face $\mathrm{C}$ so that $\mathrm{B}^{\prime}$ accurately bisects the dihedral angle between $\mathrm{C}$ and $\mathrm{C}^{\prime}$.

\subsection{Description of Oven}

After the prism components are cemented together and all excess cement removed, they are placed in a padded V-block, to be described later. The V-block with the prism components $P_{1}$ and $P_{2}$ is then placed in an electrically heated and thermostatically controlled oven, illustrated in figure 3 . The adjusting screws attached to the $V$-block protrude through the walls of the oven and are accessible to the operator from outside. A window in the top of the oven permits visual inspection of the prism at all times.

3 J. B. Saunders, Refractivity measurements on Canada balsam by interferometry, J. Research NBS 53, 373 (1954) R P2555.

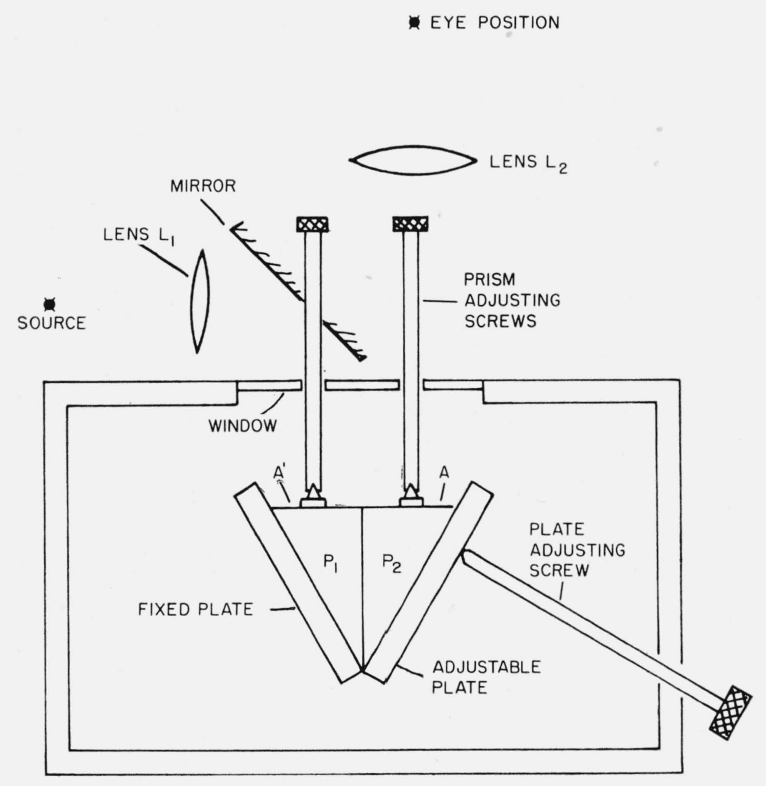

FIgUre 3. Oven, showing illuminating optics and V-block arrangement.

\subsection{Working Temperatures}

The procedure used in the cementing of the two components of the prism is that normally used in cementing lenses and prisms. An important consideration during this cementing is to avoid getting dust or other foreign particles and air bubbles into the cement. No adjustment considerations are used in the initial cementing, except to leave faces $\mathrm{A}$ and $\mathrm{A}^{\prime}$ approximately coplanar.

The adjustment of the prism components relative to each other is performed at a temperature (about $80^{\circ} \mathrm{C}$ for the cement used here) at which the viscosity of the cement is small. The prisms are adjusted at this temperature to approximate, as closely as possible, the desired condition. The temperature is then reduced to about $55^{\circ} \mathrm{C}$, where the viscosity of the cement is high. Further fine adjustments may then be completed by applying localized pressures over longer periods of time. The prism is then cooled slowly to room temperature.

\subsection{Illumination}

Inspection of the prism is made by interferometric methods, to be described later. Light from the source, shown in figure 3 , passes through a collimating lens, $\mathrm{L}_{1}$, and is reflected through the window of the oven by a mirror into prism $\mathrm{P}_{1}$. After multiple reflections in the two prisms, the light emerges from prism $\mathrm{P}_{2}$ as two coherent beams of interfering light. The observer's eye, located above lens $\mathrm{L}_{2}$, sees interference fringes in the prisms.

A line source, such as a low-pressure, unfiltered mercury-vapor lamp, is used for the preliminary adjustment of the prisms and an ordinary tungsten lamp is used for the final adjustments.

\subsection{The Adjustable V-Block \\ a. Adjusting Screws}

The V-block, illustrated in figure 4 , with its seven adjustable screws, permits the operator to make all

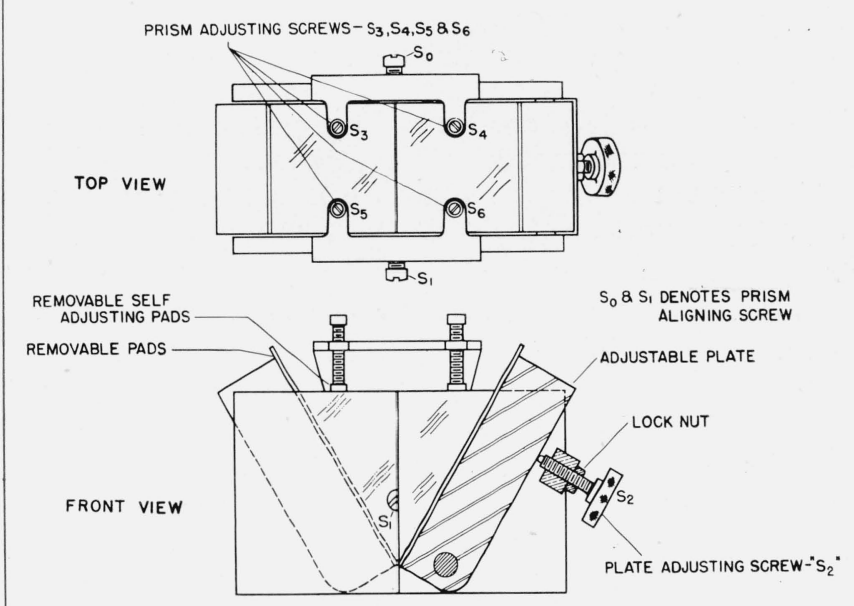

FIGURE 4. V-block and adjusting screws. 
necessary adjustments of the prisms relative to each other. There are 6 degrees of motion available -3 rotations and 3 displacements - about and along 3 rectangular coordinate axes, which lie in and normal to the dividing plane of the prism.

The prism is protected from fracture, during adjustments, by means of pads made from thick blotting paper. The cemented prism is placed in the V-block, with faces $\mathrm{C}$ and $\mathrm{C}^{\prime}$ resting on these pads. Pads are also cemented to the bottoms of the pivots that rest on faces $\dot{A}$ and $\dot{A}^{\prime}$ and through which screws $\mathrm{S}_{3}, \mathrm{~S}_{4}, \mathrm{~S}_{5}$, and $\mathrm{S}_{6}$ apply stresses to the prisms.

The effective length of the trough in the V-block is adjusted, by means of screws $S_{0}$ and $S_{1}$, to fit the length of the prism. These screws also permit the operator to move the prism along an axis parallel to the vertex of the $V$-block, thereby changing the points of contact of application of stresses by screws $\mathrm{S}_{3}$, $\mathrm{S}_{4}, \mathrm{~S}_{5}$, and $\mathrm{S}_{6}$. However, they are usually adjusted to center the prism with respect to these screws and remain fixed thereafter.

The wedge of the $\mathrm{V}$-block is controlled by screw $\mathrm{S}_{2}$. This wedge should approximate $2 \alpha$, the vertex angle of the prism, in order to avoid the formation of vapor pockets (bubbies) in the cement. If it differs too much from the angle $2 \alpha$ the prism components will become separated when stresses are applied to faces $\dot{A}$ and $A^{\prime}$. This serew permits direct control of the vertical component of the wedge in the optical cement. Prolonged application of stresses by $\mathrm{S}_{2}, \mathrm{~S}_{3}$, $\mathrm{S}_{4}, \mathrm{~S}_{5}$, and $\mathrm{S}_{6}$ causes a thinning of the film of cement and changes in the vertical wedge of the cement until it becomes equal to the difference between the wedge of the $V$-block and $2 \alpha$. Thus, the prisms are rotatable, with respect to each other, about an axis normal to the plane of figure 2 .

The horizontal component of the wedge in the cement is controlled by moving down on screws $\mathrm{S}_{3}$, $\mathrm{S}_{4}$ and up on screws $\mathrm{S}_{5}, \mathrm{~S}_{6}$ or vice versa. This represents a rotation of the prisms about an axis normal to faces $A$ and $\dot{A}^{\prime}$. Displacement along this axis is obtained by moving down on screws $\mathrm{S}_{3}, \mathrm{~S}_{5}$ and up on screws $\mathrm{S}_{4}, \mathrm{~S}_{6}$ or vice versa. Thus, one prism may be raised or lowered, relative to the other.

The prisms are rotated, relative to each other, about an axis normal to faces $\mathrm{B}$ and $\mathrm{B}^{\prime}$ by moving down on screws $\mathrm{S}_{4}, \mathrm{~S}_{5}$ and up on screws $\mathrm{S}_{3}, \mathrm{~S}_{6}$.

\section{b. Sensitivity of Adjustments}

The sensitivity of these six movements depends upon the coarseness of the screw threads and the viscosity of the cement. The latter of these may be adjusted by temperature controls over a very broad range. The sensitivity of these adjustments exceeds that of any other interferometric adjustments known to this author.

\section{Interferometric Observation of Prism for Adjustments}

In order to apply the adjustments to the required precision, the operator must use the prism surfaces as interferometers. Two interferometer arrange-
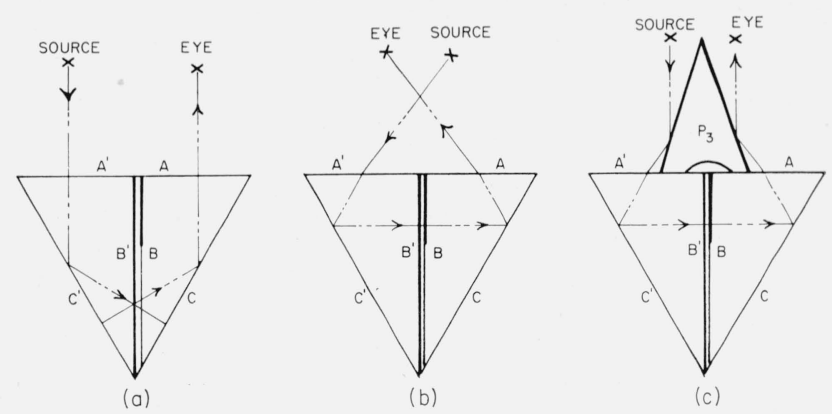

Figure 5. Interferometers in the prism.

a, Michelson-type interferometer; b and c, Fabry-Perot-type interferometer.

ments are used to adjust the prisms relative to each other, and a third is used by the optician to figure the unfinished surfaces, $\mathrm{A}$ and $\mathrm{A}^{\prime}$, and to accurately adjust the angle $\gamma$ to $90^{\circ}$

A Michelson-type interferometer is composed of the beam dividing plane $\mathrm{B}^{\prime}$ (fig. 5, a), with the two surfaces $\mathrm{C}$ and $\mathrm{C}^{\prime}$ serving as end mirrors. Light from the source above $\mathrm{A}^{\prime}$ is reflected from $\mathrm{C}^{\prime}$ to $\mathrm{B}^{\prime}$, where it divides into two equal coherent components. The reflected and transmitted components are reflected normally from $\mathrm{C}^{\prime}$ and $\mathrm{C}$, respectively, and return to plane $\mathrm{B}^{\prime}$ from which two interfering beams reach the eye of the observer above $\mathrm{A}$ after total internal reflection from $\mathrm{C}$. This interferometer is used for all adjustments of the prisms, relative to each other, except those for paralleling the film of cement.

A Fabry-Perot type of interferometer is used for paralleling and to maintain parallelism of the film of cement. It is composed of the two adjacent aluminized areas of $B$ and $B^{\prime}$. Light from the line source shown in figure $5, \mathrm{~b}$, passes through face $\mathrm{A}^{\prime}$, is reflected from $\mathrm{C}^{\prime}$ and is incident normally upon $\mathrm{B}^{\prime}$ and $\mathrm{B}$. After multiple reflection between $\mathrm{B}$ and $\mathrm{B}^{\prime}$, the several resultant beams, proceeding to $\mathrm{C}$, are reflected from $\mathrm{C}$ to $\mathrm{A}$, through which they proceed to the eye of the observer. These fringes are the narrow Tolansky ${ }^{4}$ type that results from multiple reflection. By using an isosceles prism, $\mathrm{P}_{3}$, shown in figure 5 ,c, with its surfaces made highly reflecting and placed symmetrically on face $\mathrm{AA}^{\prime}$, the FabryPerot fringes may be observed from the same position as for the Michelson interference fringes. If the Kösters prism is made of $30^{\circ}-60^{\circ}-90^{\circ}$ components, the acute angle of this isosceles prism will be $\sin ^{-1}$ $(1 / 2 n)$, where $n$ is the index of refraction of the Kösters prism.

The interferometer arrangement used by the optician to complete the prism, after the components are properly adjusted relative to each other, is shown in figure 6 . If the surfaces $A$ and $A^{\prime}$ are to be plane and coplanar, the arrangement is that of figure 6 , a. If the surfaces $\mathrm{A}$ and $\mathrm{A}^{\prime}$ are to be spherical, figure $6, b$, is the arrangement. Either white or monochromatic light may be used for this test.

${ }^{4} \mathrm{~S}$. Tolansky, Multiple-beam interferometry of surfaces and films (Clarendon Press, Oxford, 1948). 

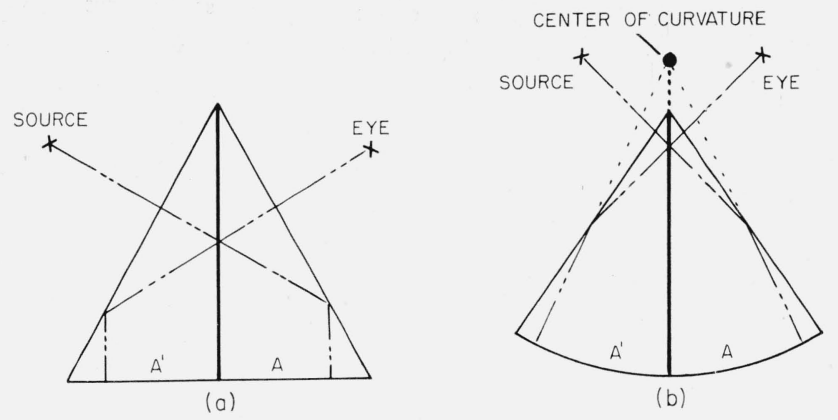

Figure 6. Interferometers in the prism.

a, Modified Michelson-type interferometer; b, modified Williams-type interferometer.

\subsection{Interpretation of Interferograms}

In order to correlate the actions of the adjusting screws wth corresponding changes in the interference fringes, the operator must be able to interpret the interferograms. These interferograms indicate the direction of the wedge in the cement and the relative orientation of the prisms.

The horizontal wedge in the cement can only be observed with the Fabry-Perot interferometer, shown in figure 5,b. This interferometer arrangement is used to eliminate the horizontal wedge and to reduce the vertical component of this wedge to a small value. The Michelson-type interferometer of figure 5,a, is used for the final adjustment of the vertical component of the wedge in the cement and for adjusting the position of one prism relative to the other. The fringes seen in this interferometer may be assumed to be due to reflection from $\mathrm{C}_{1}$ and $\mathrm{C}_{1}{ }^{\prime}$ (fig. 7), which are images of end mirrors $\mathrm{C}$ and $\mathrm{C}^{\prime}$, respectively.

* To illustrate the correlation of prism movement with changes in the interferogram, consider figure 7 . The component of the angle between $\mathrm{C}$ and $\mathrm{C}^{\prime}$, in the plane of figure 7 , indicates a vertical component in the wedge of the cement. We wish to determine

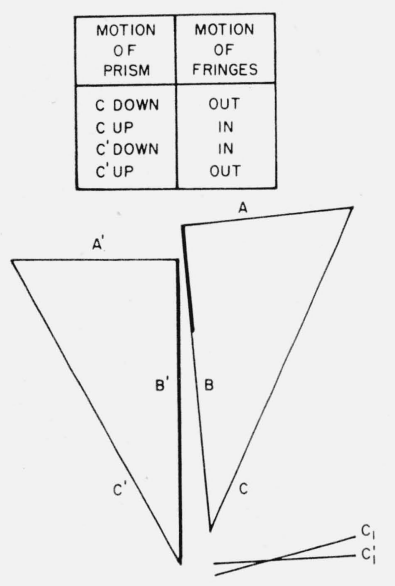

(a)

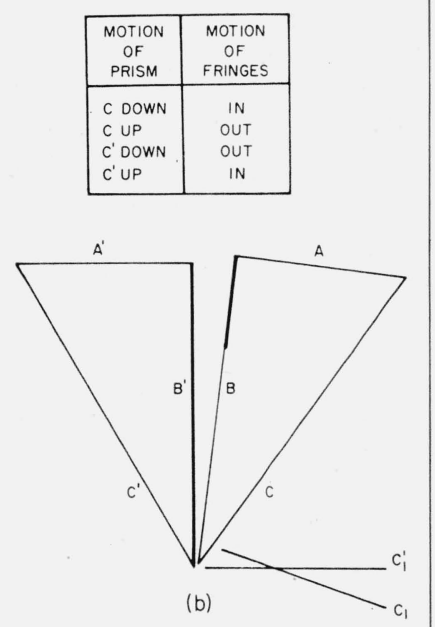

Figure 7. Diagram and table for interpretation of fringes. the direction of this wedge in order to know how to change it. The combinations, "motion of prism" with "motion of fringes," tabulated for each of the two conditions, enable the operator to determine which condition applies to the prism being adjusted. When prism $\mathrm{ABC}$ is moved down, for instance, $\mathrm{C}_{1}$ moves down relative to $\mathrm{C}_{1}^{\prime}$, causing a lateral movement of their point of intersection where the zeroorder fringe is located. Because all the fringes move in this direction, the zero-order fringe need not be visible. The direction of motion of the fringes, along a line normal to $\mathrm{B}$ or $\mathrm{B}^{\prime}$, indicates the direction of the wedge in the cement.

After determination of the direction of the vertical component of the wedge in the cement, the operator adjusts screw $S_{2}$ to correct it. The author keeps a copy of figure 7 before him at all times during the adjustment operations.

\subsection{Considerations Necessary for Making Prism Adjustments}

The choice of the adjustment desired depends upon the intended application of the prism. Four types of finished adjustments have been used at the Bureau. These are illustrated in figure 8 . The fringes of figure 8 , a, have a most favorable width (assumed here to be one-fifth the aperture of the prism) for ease of reading and are parallel to the dividing plane of the prism. In figure $8, b$, the fringes have a most favorable width and are adjusted to be perpendicular to the dividing plane. Figure $8, c$, shows a pattern produced by a perfectly symmetrical prism. The order of interference is zero over the entire field of interference. Because the order of interference in the finished prism remains unchanged along the margin that is adjacent to the dividing plane, the zero-order fringe is confined to this margin during adjustments in later applications. If it is desired to have the zero-order fringe at the center of the field when the fringes are parallel to the dividing plane and of a most favorable width, the fringe pattern should be adjusted to that shown in figure 8,d. The zero-order fringe, in all other cases, is adjusted to pass through the center of the aperture.

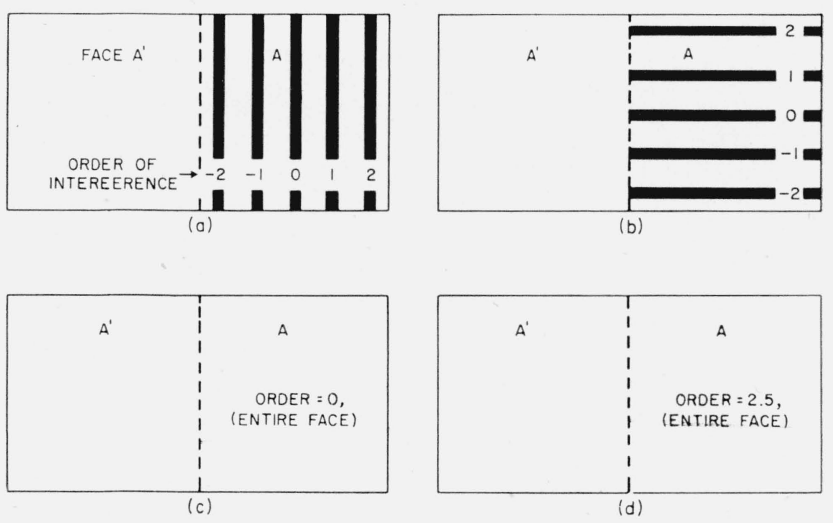

Figure 8. Diagrammatic representation of several prism adjustments. 
The wedges that are indicated by the fringes in figure 8 ,a, and 8 ,b, are "built-in wedges." When the prism is used in combination with other elements, the built-in wedge is added to that obtained by adjustments of the apparatus, such as tilting of end mirrors, or to the wave-front aberrations in the auxiliary optics of the system. Thus, when a prism with a built-in wedge is used for testing nearly perfect optical systems, the fringes never become too broad to measure fractions of fringes.

\subsection{Adjustment Procedure}

When the prism is placed in the $V$-block and in the oven, it will usually not present visible interference fringes. A small object (the sharp point of a sewing needle is excellent) is placed on surface $\mathrm{A}^{\prime}$, indicated by $\mathrm{X}$ in figure 2,a. On looking into face $\mathrm{A}$ from above, two images $\left(\mathrm{X}_{1}\right.$ and $\left.\mathrm{X}_{2}\right)$ of $\mathrm{X}$ will be seen. The separation of these two images may be reduced to zero by manipulation of the five adjusting screws. If screws $\mathrm{S}_{3}$ and $\mathrm{S}_{6}$ of figure 4 are moved down (or up), the images of $\mathrm{D}$ move in opposite directions parallel to a line through $\mathrm{S}_{4}$ and $\mathrm{S}_{6}$. If $\mathrm{S}_{2}$ is changed while the other screws are applying some pressure, the images move in opposite directions along a line perpendicular to the plane of B. A low-power telescope, or long-focus microscope, permits improved vision of these images. When the images appear close together, interference fringes can be seen if a homogeneous source is used. The separation of the fringes is inversely proportional to the separation of the images of D.

Experience indicates that the best procedure for adjusting the Kösters prism is as follows:

(a) Adjust the film of cement to the desired wedge by means of the Fabry-Perot interferometer, described above.

(b) Adjust the two refracting edges, that form the vertex of the angle $\alpha$ (fig. 2), to the desired value by rotating one prism relative to the other.

(c) Adjust the zero order of interference to the desired position by vertical adjustments of the prism.

Operations (a) and (b) determine the width and orientation of the fringes, and operation (c) determines the order of interference.

(d) Reduce the temperature of the oven to $51^{\circ} \mathrm{C}$ and straighten the fringes by local application of pressures. The position of the zero-order fringe is again corrected and the prism cooled to room temperature.

(e) Grind, polish, and adjust by altering the shape of face $\mathrm{AA}^{\prime}$ of the prism so that the fringes seen in the interferometer of figure 6 are similar to those seen in the interferometer of figure 5,a. When these two sets of fringes are identical, angle $\gamma$ is precisely $90^{\circ}$; or in case surface $\mathrm{AA}^{\prime}$ is made spherical, the center of curvature lies in the dividing plane of the prism.

\subsection{Adjustment for White-Light Fringes}

If a white-light source is placed behind the line source the fringes tend to fade into the brighter white background. However, they will remain sufficiently visible to permit adjustments if the intensity of the white-light source is kept sufficiently low. The prism surface, $\mathbf{A}$ or $\mathbf{A}^{\prime}$, which appears highest by mechanical test, will, in general, need to be lowered in order to decrease the order of interference. This prism is lowered, by means of the prism adjusting screws, while maintaining fringes of sufficient width to detect the zero band when it passes across the field. An increase in contrast indicates a lowering of the order of interference. The fringes near the zero order will be brilliantly colored and quite visible.

\subsection{Final Correction}

If the prisms are large, the fringes will be difficult to straighten. This curvature of the fringes is due to incomplete relaxation of stresses in the prism, caused by the effective high viscosity of liquids when in the form of very thin films. Leaving the film of cement thick favors this relaxation. An experienced operator, however, can improve on the straightness of the fringes by a form of kneading. This is done by local distribution of the areas of the supporting pads and localized application of forces. The details of this operation are too extensive to be given here. It is more easily effected, however, at temperatures at which the cement is highly viscous but soft enough to be kneaded by prolonged application of unbalanced forces. The author finds $55^{\circ} \mathrm{C}$ to be a favorable working temperature for this operation with Canada balsam that is treated to have a consistency considered proper for such use. The prism is adjusted and allowed to relax while holding at $80^{\circ} \mathrm{C}$ for a prolonged period ( $1 \mathrm{hr}$ or more). It is then cooled slowly to $55^{\circ} \mathrm{C}$ and allowed to remain at this temperature over night, with all adjusting screws loosened and the V-block properly adjusted. This overnight wait is used because 1 working day does not allow sufficient time to complete the adjustments. Sometimes 3 or more days may be required to attain the perfection required in the fringe pattern. It is usually sufficient to straighten the fringes in the central one-half of the aperture, ignoring the curvature of the fringes in the marginal regions. However, there are applications of these prisms for which it is desirable, although not necessary for precision results, to have the three lowest-order fringes (the zero order and its two neighbors) straight from end to end. When the fringes are adequately straight and the zero order is properly positioned, all adjusting screws are relaxed and the prism allowed to cool slowly to ambient temperatures.

\subsection{Finishing of Prism}

Large prisms will require reinforcement to prevent relative shifting of the components at ambient temperatures and over long periods of use, if Canada balsam is used for cement. The ends of the two prisms will usually not be coplanar when the above adjustments are completed. The two screws, $S_{0}$ and $S_{1}$, shown in figure 4 , prevent excessive lateral shifting of the prisms along the $\mathrm{V}$-block. After the 


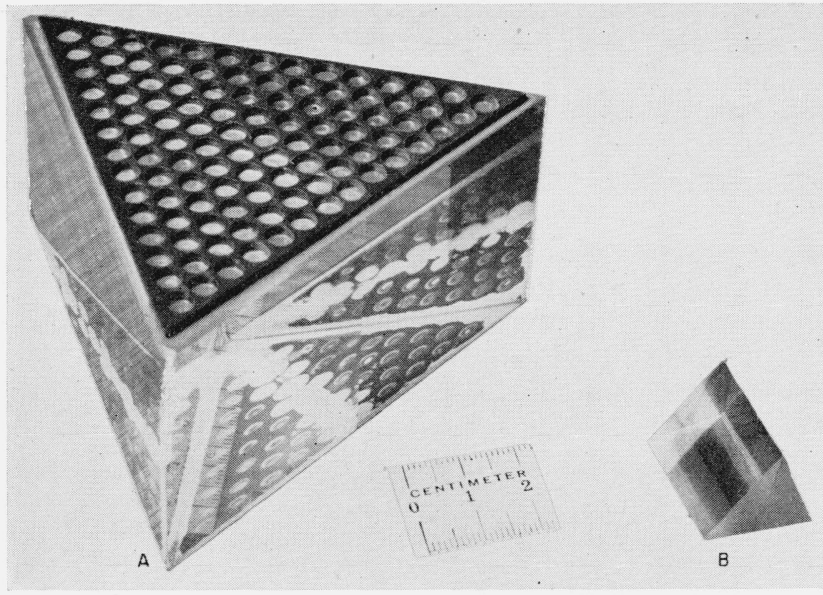

FiguRe 9. Finished prisms.

a, Kösters prism, $30^{\circ}-60^{\circ}-90^{\circ}$ elements; b, Kösters prism, $33^{\circ}-57^{\circ}-90^{\circ}$ elements

prism is removed from the furnace, the two ends are ground until plane and approximately parallel. A perforated triangular plate of steel (fig. 9, a) is then cemented over each of the two ends, avoiding contact between the adhesive and the edges of the film of optical cement. This prevents contamination of the optical cement, and the perforations allow the adhesive to dry out evenly over the entire plate. Steel is used because its coefficient of thermal expansion approximates that of the glass more closely than other metals that can be used. Figure 9 is a photograph of two finished prisms. Figure 9, a, is a typical Kösters prism with $30^{\circ}-60^{\circ}-90^{\circ}$ elements, and figure $9, \mathrm{~b}$, is a smaller prism with $33^{\circ}-57^{\circ}-90^{\circ}$ elements. The base of the small prism is intended to be finished spherically and the edges ground cylindrically to fit in a 1 -in. eyepiece tube.

The end plates in figure 9 , a, are shaped and cemented in place so that they do not interfere with the final polishing of the base of the prism. The use of end plates may be avoided by using thermosetting cement. The prism of figure $9, \mathrm{~b}$, is too small to require end-plate reinforcement.

The base of the prism is ready for its final finish when the end plates are on and the adhesive inured. Initially, a small flashlight lamp is used until the two images of it, seen in the prism, are brought into approximate coincidence by successive grinding, polishing or buffing, and testing. An extended white-light source may then be used for the remaining tests if the prisms have previously been adjusted to the conditions shown in figures $8, \mathrm{a}, \mathrm{d}$, or $\mathrm{c}$. The shape of the fringes, observed in this test, is a measure of the symmetry of the prism, which includes equality of the angles. The flatness of the Kösters prism base may be tested against a master flat by conventional optical-shop methods, and the sphericity of the spherical base can be tested against a

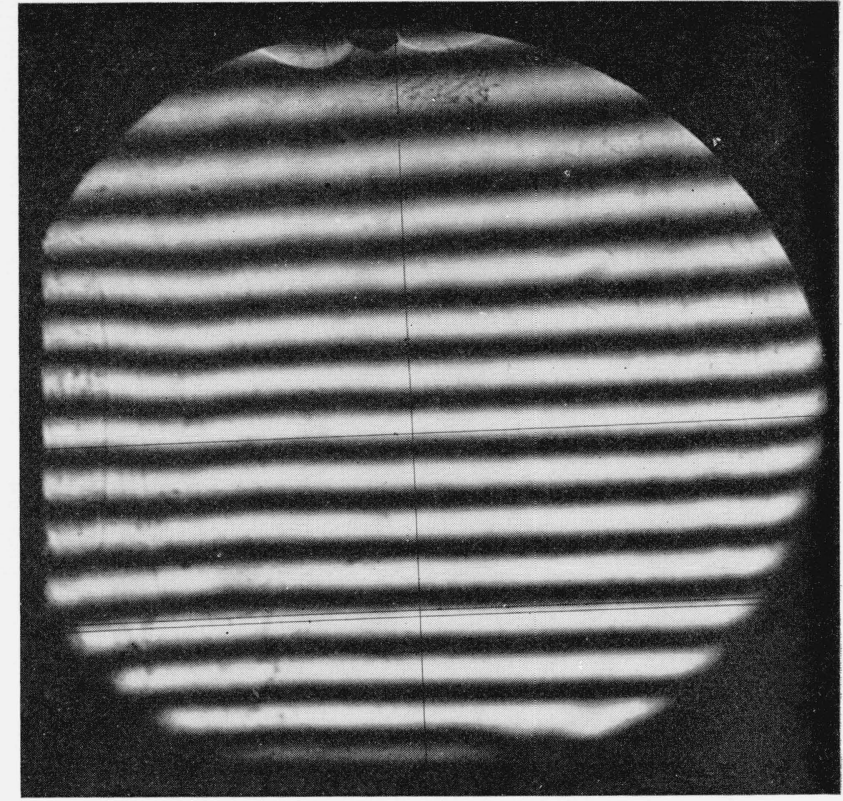

FIGURE 10. Fringe pattern produced by a cemented Kösters double-image prism.

matched test plate; however, its perfection is not so critical as to require the added cost of a test plate. An experienced optician can usually make this surface sufficiently nearly spherical for all practical applications currently anticipated. A Foucault knifeedge test may be used, with limited precision, even though refraction at faces $\mathrm{C}$ and $\mathrm{C}^{\prime}$ deforms the wave front.

The Kösters prisms supplied by one manufacturer have angles of $30^{\circ} 30^{\prime}, 88^{\circ} 30^{\prime}, 61^{\circ}$, and are attached with cedar-wood oil. 'This choice of angles is used to eliminate ghost images from the prism surfaces. To eliminate the ghost images from a $30^{\circ}-60^{\circ}-90^{\circ}$ prism it is only necessary to tilt the prism slightly about an axis perpendicular to the dividing plane. This manner of eliminating reflected light avoids differential diffraction at faces $\mathbf{A}$ and $\mathbf{A}^{\prime}$.

Figure 10 shows a fringe pattern produced by one of the Kösters double-image-type prisms.

\section{Conclusion}

The methods for constructing and adjusting prisms of the Kösters type, outlined here, permit the production of high-precision prisms. Interferometric procedures are described whereby all tests are easily and accurately performed with no auxiliary apparatus other than light sources. Several prisms have been constructed and adjusted at the Bureau and, so far, have proved quite stable.

Washington, August 31, 1956. 